\title{
EFEITO DE ÓLEOS ESSENCIAIS SOBRE A MICOFLORA ASSOCIADA ÀS SEMENTES DE MANJERICÃO
}

\author{
Ronice Alves Veloso ${ }^{1}$, Evelynne Urzedo Leão ${ }^{2}$, Dalmárcia de Souza Carlos Mourão ${ }^{3}$, Talita Pereira \\ de Souza Ferreira ${ }^{4}$, David Ingsson Oliveira Andrade de Farias ${ }^{5}$, Gil Rodrigues dos Santos ${ }^{6}$
}

\section{RESUMO:}

A escassa aplicação de controle fitossanitário às espécies medicinais, como o manjericão, torna as suas sementes em importantes veículos de propagação de fitopatógenos. Neste sentido, objetivou-se avaliar o efeito de óleos essenciais sobre a incidência de fungos nas sementes de manjericão utilizando diferentes concentrações de óleos essenciais. Adotou-se o delineamento inteiramente casualizado com quatro repetições, em esquema fatorial 3 x 10 (óleo essencial de manjericão, capim citronela e copaíba e dez concentrações dos óleos essenciais: $0 ; 5 ; 10 ; 15 ; 20 ; 25 ; 30 ; 35 ; 40$ e 100 \% v.v). Foram realizados testes com os óleos essenciais para avaliar o efeito sobre a germinação das sementes e a inibição da micoflora presente nas sementes de manjericão pelo Blotter test; e posteriormente, o teste de patogenicidade. Foram identificados seis gêneros de fungos, sendo alguns causadores de manchas foliares, como Curvularia sp.; Colletotrichum sp. e Alternaria sp., e os demais gêneros, como Aspergillus sp., Cladosporium sp. Penicillium sp., considerados fungos contaminantes de armazenamento. Observou-se inibição da germinação das sementes com o aumento das concentrações dos óleos essenciais.

Palavras-chave: Ocimum basilicum, patologia de sementes, germinação.

\section{EFFECT OF ESSENTIAL OILS ON MYCOFLORA ASSOCIATED WITH BASIL SEEDS}

\begin{abstract}
:
The scarce application of phytosanitary control to the medicinal species, such as basil, makes its seeds an important vector for the propagation of phytopathogens. In this sense, the objective of this study was to evaluate the effect of essential oils on the incidence of fungi in basil seeds using different concentrations of essential oils. A completely randomized design was adopted with four repetitions, in a $3 \mathrm{x}$ 10 factorial scheme (basil essential oil, citronella grass and copaíba and ten concentrations of essential oils: $0 ; 5 ; 10 ; 15 ; 20 ; 25 ; 30 ; 35 ; 40$ and $100 \% \mathrm{vv})$. Tests with essential oils were carried out to evaluate the effect on seed germination and the inhibition of mycoflora present in basil seeds by the Blotter test; and subsequently, the pathogenicity test. Six genera of fungi were identified, some causing leaf spots, such as Curvularia sp..; Colletotrichum sp. and Alternaria sp., and the other genus, such as Aspergillus sp.,
\end{abstract}

\footnotetext{
${ }^{1}$ Eng. Agrônoma, Dra. em Produção Vegetal (Universidade Federal do Tocantins), Gurupi - TO. ronicealves@ hotmail.com;

${ }^{2}$ Eng. Agrônoma, Dra. em Agronomia (Proteção de Plantas) (Universidade Estadual Paulista Júlio de Mesquita Filho), São Paulo - SP. evelynnegpi@hotmail.com;

${ }^{3}$ Téc. Laboratório, Doutoranda em Produção Vegetal (Universidade Federal do Tocantins), Gurupi - TO. dalmarciaadm@uft.edu.br;

${ }^{4}$ Eng. de Bioprocessos e Biotecnologia, Dra. em Biotecnologia (Universidade Federal do Tocantins), Gurupi - TO. talitapsf@uft.edu.br;

${ }^{5}$ Eng. Agrônomo, Doutorando em Produção Vegetal (Universidade Federal do Tocantins), Gurupi - TO. david_ingsson@hotmail.com;

${ }^{6}$ Eng. Agrônomo, Dr. em Fitopatologia (Universidade de Brasília), Brasília - DF. gilrsan@uft.edu.br.
} 
Revista Agri-Environmental Sciences, Palmas-TO, v. 6, e020004, 2020

ISSN 2525-4804

Cladosporium sp. Penicillium sp., considered contaminating storage fungi. Results showed that the germination of the seeds was inhibited as the concentration of essential oils was increased.

Keywords: Ocimum basilicum, seeds pathology, germination. 


\section{INTRODUÇÃO}

O manjericão (Ocimum basilicum L.) é uma espécie herbácea, pertencente à família Lamiaceae, originário do Sudoeste Asiático e da África Central. Este foi introduzido no Brasil pelos colonos italianos, adaptando-se bem às condições climáticas brasileiras, podendo ser cultivado o ano todo. Apresenta grande valor econômico, com utilização para diversos fins, como ornamental, condimentar, medicinal, aromática, na indústria farmacêutica e de cosméticos e para produção de óleo essencial (Blank et al., 2010; Rosado et al., 2011). Além disso, estudos têm demonstrado que o óleo essencial de manjericão apresenta propriedades inseticidas, repelentes e antimicrobianas, podendo ser empregado também na conservação de grãos (Fernandes et al., 2004; Flávio et al., 2014).

De modo geral, e com o aumento do cultivo da espécie, tornou-se inevitável o surgimento de problemas fitossanitários, principalmente fungos, os quais, além de ocasionarem perdas na produção, podem, ainda, provocar alterações nos compostos químicos da planta e reduzir suas propriedades terapêuticas. As doenças fúngicas mais frequentes em plantas medicinais como o manjericão são: antracnose, ferrugem, carvão, podridão, oídio, míldio e murcha (Kruppa e Russomano, 2008).

A qualidade sanitária das sementes, por exemplo, é um dos aspectos mais importantes relacionados à produção em razão do grande número de microrganismos patogênicos que podem estar associados a elas. Microrganismos podem causar anormalidades e lesões nas plântulas, bem como deterioração do tecido embrionário, comprometendo a germinação e vigor das sementes e consequentemente perdas de produção (Moreau, 2011; Piveta et al., 2010). Há relatos de fungos patogênicos transmitidos por sementes de plantas da família Lamiaceae, como o manjericão, prejudicando o desenvolvimento de plântulas, causando manchas foliares ou murchas (Urben et al., 1987; Mendes et al., 1998; 2003; Kruppa \& Russomanno, 2001; Russomanno et al., 2004).
Neste sentido, o controle de doenças em espécies medicinais, condimentares e aromáticas não é uma atividade simples a ser realizada, uma vez que a utilização de agrotóxicos pode comprometer a produção e composição química dos princípios ativos de interesse econômico (Russomanno e Kruppa, 2010). No Brasil, o controle fitossanitário de doenças em espécies de plantas medicinais não é realizado de forma rigorosa. Assim, a alternativa tem sido a utilização de sementes sadias de alto vigor, mudas e materiais propagativos livres de quaisquer tipos de patógenos e provenientes de fornecedores idôneos, evitando dessa maneira a introdução de microrganismos patogênicos em novas áreas de cultivo (Russomanno e Kruppa, 2010; Kruppa e Russomano, 2011).

A utilização de extratos (óleos fixos) e óleos essenciais (voláteis) de plantas medicinais, condimentares e aromáticas com potencial para o manejo de doenças de plantas apresenta-se atualmente como alternativa ao uso de agrotóxicos em diversas culturas (Medice et al., 2007; Seixas et al., 2011; Garcia et al., 2012; Sarmento-Brum et al., 2013). Isso possibilita sistemas de cultivo menos dependentes ao uso de agrotóxicos e são mais sustentáveis (Seixas et al., 2011). Óleos essenciais são estruturas complexas sintetizadas a partir do metabolismo secundário de algumas espécies vegetais (Miranda et al., 2016). Sua essência consiste em elementos voláteis contidos em diversos órgãos da planta e estão relacionados a funções necessárias a sobrevivência vegetal, exercendo papel fundamental na defesa contra microrganismos e predadores (Ribeiro et al., 2012; Ootani et al., 2013). Óleos essenciais de plantas como a copaíba (Copaifera langsdorffii L.), capim citronela (Cymbopogon nardus L.), manjericão, alecrimpimenta (Lippia sidoides Cham.), capim-limão (Cymbopogon citratus), noni (Morinda citrifolia L), entre outros, já foram relatados como potenciais para o manejo de doenças de plantas (Lopes et al., 2013; Silva et al., 2016; Sales et al., 2018).

No contexto científico, estudos avaliando o efeito de óleos essenciais sob a micoflora fúngica 
de sementes de manjericão são inexistentes ou escassos. Frente a isso, objetivou-se avaliar o efeito de óleos essenciais sobre a incidência de fungos nas sementes de manjericão em função de suas diferentes concentrações.

\section{MATERIAL E MÉTODOS}

Os ensaios foram conduzidos no Laboratório de Fitopatologia da Universidade Federal do Tocantins, Campus de Gurupi, município de Gurupi, TO. As sementes de manjericão utilizadas foram adquiridas no comércio local, em embalagem de $50 \mathrm{~g}$ (ISLA SEMENTES), com percentual de germinação e pureza de 85 e $100 \%$, respectivamente. As sementes foram conservadas em embalagem original e mantidas a $25^{\circ} \mathrm{C}$ em refrigerador.

Utilizaram-se 200 sementes no ensaio da inibição da microflora e avaliação da germinação. A análise da sanidade das sementes e a aplicação do controle com os óleos essenciais foram realizadas pelo método Blotter test (Brasil, 2009). Para avaliar a inibição do crescimento micelial dos fungos presentes nas sementes de manjericão foram implantados ensaios em delineamento experimental inteiramente casualizado em esquema fatorial $3 \times 10$ (três tratamentos $\mathrm{x}$ dez concentrações), com quatro repetições, sendo cada repetição uma caixa de plástico transparente (gerbox) com 50 sementes. Os tratamentos utilizados foram: óleo essencial de manjericão, de capim citronela e de copaíba. Para todos os óleos essenciais foram utilizadas as seguintes concentrações: $0 ; 5 ; 10 ; 15 ; 20 ; 25 ; 30 ; 35$; 40 e $100 \%$ (v/v).

As concentrações de cada óleo essencial foram preparadas utilizando, como agente dispersante Tween $^{\circledR} 80$ a $0,5 \%$. Primeiramente, preparou-se a solução de óleo essencial à concentração de $100 \%$, a qual foi submetida a diluições para obtenção das demais concentrações de óleo essencial. As sementes de manjericão previamente esterilizadas foram tratadas com $0,5 \mathrm{~mL}$ de cada concentração dos óleos essenciais através de imersão, e foram dispostas em caixas gerbox forradas com duas folhas de papel germitest umedecido com $10 \mathrm{~mL}$ de água destilada estéril.

Em seguida, as caixas gerbox foram mantidas em câmara de incubação a $25{ }^{\circ} \mathrm{C} \pm 2{ }^{\circ} \mathrm{C}$ e fotoperíodo de 12 horas, por sete dias. Após o período de incubação, avaliou-se a germinação e a incidência de fungos associados às sementes, os quais foram expressos em percentagem. Após observação das estruturas fúngicas, em microscópio estereoscópico e óptico, os fungos foram identificados com auxílio de literatura especializada (Ellis, 1971; Barnett e Hunter, 1972).

Os dados obtidos quanto à germinação de sementes e inibição da micoflora foram avaliados e as médias obtidas foram submetidas à análise de regressão, e demonstradas em gráficos com os valores do $\mathrm{R}^{2}$ e Equação.

Os fungos encontrados no Blotter test foram isolados em placas de Petri, contendo meio de cultura Batata Dextrose Ágar (BDA). Posteriormente, foram inoculados em plantas sadias de manjericão para verificar a patogenicidade dos fungos, em condições de casa de vegetação. Plantas de manjericão, aos 30 dias após semeadura e cultivadas em vasos plásticos, foram inoculadas com $20 \mathrm{~mL}$ das suspensões de cada um dos fungos encontrados na concentração de $10^{6}$ conídios $\mathrm{mL}^{-1}$. As plantas do tratamento testemunha foram pulverizadas com água destilada, acrescida de Tween ${ }^{\circledR}$. Em seguida, as plantas foram mantidas em câmara úmida e escura à $25^{\circ} \mathrm{C} \pm 2^{\circ} \mathrm{C}$, por $24 \mathrm{~h}$. Após esse período, as plantas foram mantidas em condições de casa de vegetação $\left(30 \pm 5^{\circ} \mathrm{C}\right)$ e com irrigação diária sempre que necessário. Foram analisadas diariamente, para diagnosticar os sintomas característicos de presença de doenças.

Visando o cumprimento dos postulados de Koch, fragmentos de folhas de manjericão com lesões provenientes das inoculações foram submetidos à assepsia em soluções de álcool 50\%, hipoclorito de sódio a $1 \%$ e três lavagens em água destilada esterilizada. Em seguida, os fragmentos foliares foram depositados no centro de placas de 
Petri contendo BDA e mantidas em incubadora à $25^{\circ} \mathrm{C} \pm 2^{\circ} \mathrm{C}$, por sete dias. Após o desenvolvimento dos fungos, efetuou-se a identificação e a confirmação de sua patogenicidade. A patogenicidade foi constatada visualmente e o resultado obtido descreveu se o fungo foi patogênico ou não patogênico, não avaliando a severidade dos sintomas.

\section{RESULTADOS E DISCUSSÃO}

A partir da análise sanitária das sementes de manjericão foram identificados os seguintes gêneros fúngicos no tratamento testemunha: Alternaria sp. (3\%); Colletotrichum sp. (2\%); Curvularia sp. (1,5\%); Aspergillus sp. (3,5\%); Cladosporium sp. (6,5\%); Penicillium sp. (1\%). Os gêneros Aspergillus sp., Cladosporium sp. e Penicillium sp. foram os de maior ocorrência nas sementes de manjericão e foi possível detectá-los em todos os tratamentos com os óleos essenciais testados. Essas espécies de fungos são consideradas contaminantes em sementes de plantas medicinais, condimentares e aromáticas, sendo que a presença de Aspergillus e Penicillium indica, geralmente, má qualidade ou problemas na conservação das sementes (Kruppa e Russomanno, 2009).

Na Figura 1 estão descritos os resultados quanto à inibição da micoflora sob as diferentes concentrações de óleos essenciais. Nenhum modelo de regressão foi ajustado para a incidência de Aspergillus sp. quando submetido as diferentes concentrações dos óleos essenciais de manjericão, capim citronela e copaíba (Figura 1). Para a incidência de Aspergillus sp. e Cladosporium sp. as médias foram ajustadas ao modelo quadrático de regressão e apresentou comportamento decrescente de acordo com o incremento da concentração de óleo de manjericão e capim citronela, evidenciando um efeito deletério do óleo para estes patógenos (Figura 1AB). A partir da concentração de $30 \%$ e $40 \%$ do óleo essencial não se evidenciou crescimento fúngico nas sementes de manjericão.
Para o tratamento com as diferentes concentrações do óleo de copaíba observou-se efeitos adversos, não sendo ajustado nenhum modelo de regressão significativo. Entretanto, observou-se a inibição da incidência de fungos somente na concentração de $100 \%$, não apresentando resultados satisfatórios de inibição nas demais concentrações (Figura 1C).

Gomes et al., (2016) avaliando a eficiência de óleos essenciais na qualidade sanitária e fisiológica em sementes de feijão-fava (Phaseolus lunatus L.) observaram que os óleos essenciais de copaíba e manjericão reduziram consideravelmente o percentual de incidência de Fusarium sp. e Cladosporium sp. associados as sementes de feijãofava, resultado semelhante observado neste estudo para o gênero Cladosporium sp.

Observou-se inibição da germinação das sementes com o aumento das concentrações dos óleos essenciais, quando comparado como o tratamento testemunha (Figura 2). As médias de germinação foram ajustadas ao modelo quadrático de regressão. No tratamento testemunha, o percentual de germinação foi de $88 \%$, mostrando que a germinação está dentro dos padrões informados pela empresa. A partir da concentração de $40 \%$ dos óleos essenciais de manjericão e capim citronela houve inibição total da germinação de sementes. Já o óleo essencial de copaíba apresentou efeito menos agressivo ao embrião quando as sementes foram submetidas às concentrações de $40 \%$ e $100 \%$, pois o percentual de germinação foi de $71,5 \%$ e $49 \%$, respectivamente.

O efeito de inibição da germinação pode estar relacionado com a elevada concentração dos óleos essenciais, ou a algum composto ou substância presente no mesmo capaz de impedir o processo de germinação, visto que entre os constituintes dos óleos essenciais podemos ter a presença de compostos ou combinação de compostos com efeitos alelopáticos (Hillen, et al.,2012). 

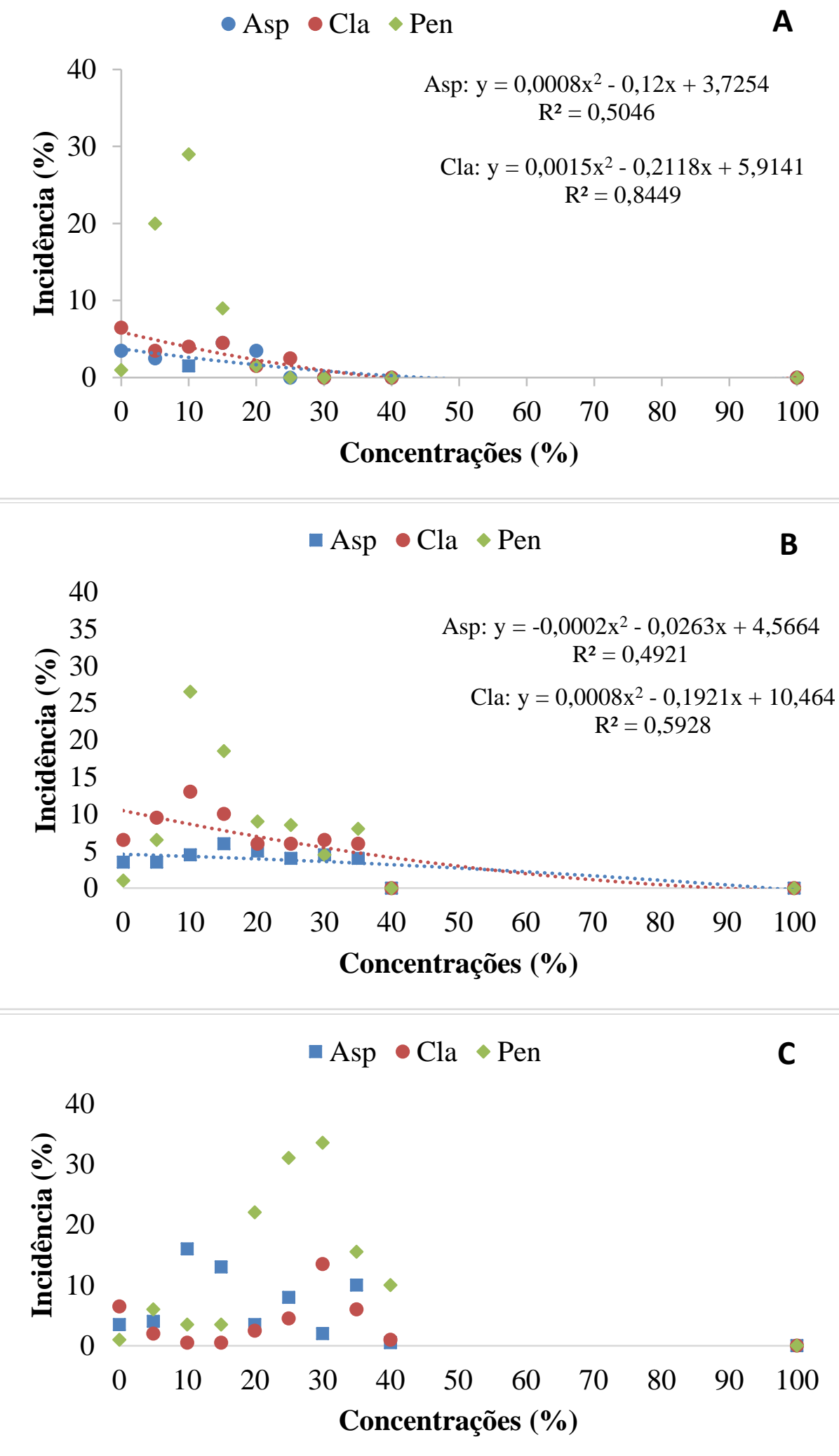

Figura 1. Valores percentuais de incidência de fungos presentes em sementes de manjericão tratadas com diferentes concentrações de óleo essencial de manjericão (Ocimum basilicum L.) (A), capim citronela (Cymbopogon nardus L.) (B) e copaíba (Copaifera spp.) (C). * Asp = Aspergillus sp., Cla = Cladosporium sp., Pen= Penicillium sp. 
Brito et al. (2010), estudando os efeitos de óleos essenciais de canela e manjericão na germinação de sementes de mandacaru (Cereus jamacaru P. DC.), observaram redução na germinação das sementes tratamento com óleo de manjericão, apresentando menor valor de massa seca e tamanho das plântulas, e interferindo negativamente no vigor das sementes de mandacaru.

A avaliação da qualidade sanitária das sementes com o emprego de extratos vegetais tem sido analisada por diversos autores, como Silva et al., (2009), Medeiros et al., (2011) e (2013). Neste estudo, a utilização de óleos essenciais na inibição da micoflora em sementes de manjericão apresentou efeito promissor, no entanto, ao elevar-se as concentrações de óleos essenciais observou-se redução no percentual de germinação das sementes.

Lima e Villela (2017), avaliando o efeito dos óleos essenciais de alho e laranja e do surfactante Tween $^{\circledR} 80$ sobre a germinação de sementes de manjericão, observaram que a imersão por um minuto em solução preparada com os óleos até a concentração de $10 \%$ pode ser realizada com segurança, sem prejuízos à germinação dessas sementes. Resultado semelhante pode ser observado neste estudo, pois as concentrações dos óleos essenciais acima de $20 \%$ proporcionam efeito deletério nas sementes. Por isso, recomendamos a utilização desse óleo sem ultrapassar esta concentração.

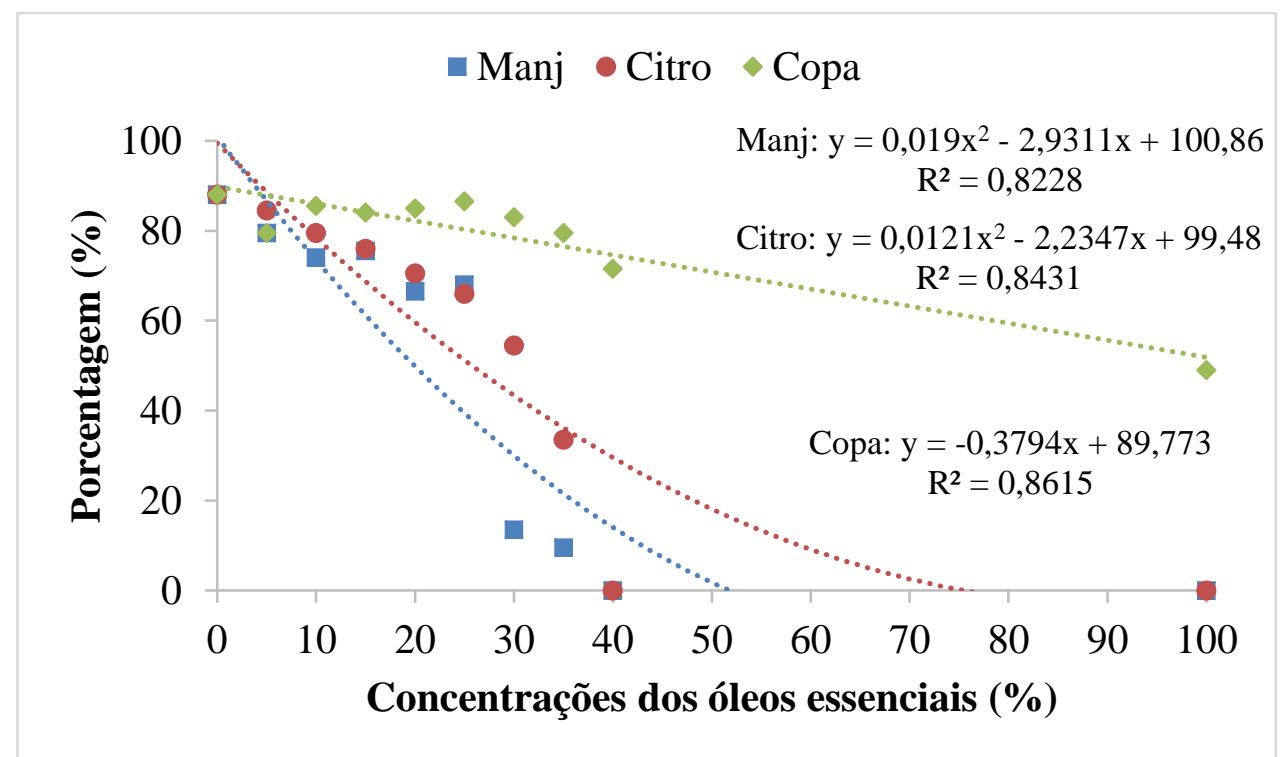

Figura 2. Porcentagem de germinação de sementes de manjericão tratadas com diferentes concentrações de óleo essencial de manjericão (Ocimum basilicum L.) (Manj), capim citronela (Cymbopogon nardus L.) (Citro) e copaíba (Copaifera spp.) (Copa).

Quanto à patogenicidade, constatou-se que os gêneros Alternaria, Colletotrichum e Curvularia provocaram o surgimento de lesões necróticas e amarelecimento do limbo foliar em plantas de manjericão. Com a evolução das lesões houve abscisão foliar e comprometimento da produção de biomassa da parte aérea (folhas e estruturas reprodutivas), a qual é o principal produto comercializado de espécies medicinais condimentares e aromáticas da família Lamiaceae, pois são utilizadas no preparo de chás, em condimentos e para a extração de óleo essencial.

A falta de medidas fitossanitárias quanto à qualidade de sementes de espécies medicinais é um fator de elevada importância na condução de cultivos em grande escala, uma vez, que as sementes são veículos de introdução de patógenos em novas áreas. Um exemplo da disseminação de patógenos 
por sementes é o relatado por Elmer (2001), onde Fusarim oxysporum f. sp. basilici agente causal da murcha de manjericão foi descrito na Rússia (1956), Itália (1970) e França (1980). Posteriormente, em 1990 foi relatada pela primeira vez nos Estados Unidos e três anos depois havia relatos da doença em mais de 25 países abrangendo três continentes.

Esta disseminação pode ter ocorrido em razão da grande demanda de sementes de manjericão para novas áreas produtoras e a falta de medidas fitossanitárias para o comércio destas sementes. Reis et al, (2007) relatam o primeiro registro formal da presença do fungo $F$. oxysporum f. sp. basilici no Brasil sugerindo que as sementes representam o mais provável veículo de introdução e potencial disseminação deste patógeno em território nacional. Em nosso estudo, a incidência do gênero Fusarium sp. foi insignificante, entretanto, precauções devem ser tomadas no comércio de sementes de manjericão, visto as perdas significativas que este patógeno pode causar.

Dessa maneira, é pertinente concluir que os óleos essenciais possam ser utilizados em tratamentos sanitários de sementes, contudo, o efeito desses óleos sobre a germinação e o desenvolvimento das plântulas precisa ser também investigado. A utilização da concentração correta, aquela que inibe a incidência de fitopatógenos e não compromete a germinação das sementes, é o ponto chave para a utilização de óleos essenciais no tratamento das sementes. Do mesmo modo, prevenir o estabelecimento de agentes patogênicos nos cultivos ainda é a melhor alternativa aos adeptos a utilização de substâncias de origem natural.

\section{CONCLUSÃO}

Os gêneros fúngicos com maior incidência nas sementes tratadas com os três tipos de óleos essenciais avaliados foram: Cladosporium sp. e Penicillium sp. Destes foi observada total ausência de germinação de sementes a partir da concentração de $40 \%$ de óleo essencial de manjericão e capim citronela.

\section{REFFERÊNCIAS}

Barnett. H.C. \& Hunter. B.B. (1972). Illustrated genera of imperfect fungi. $3^{\circ}$ ed. Mineapolis: Burgess Publishing. 241p.

Blank, A.F.; Souza, E.M.; Paula, J.W.A. \& Alves. P. B. (2010). Comportamento fenotípico e genotípico de populações de manjericão. Horticultura $\begin{array}{llll}\text { Brasileira } & 28 & \text { (3): } & 305-310 .\end{array}$ https://doi.org/10.1590/S0102-05362010000300011

Brasil (2009). Ministério da Agricultura. Pecuária e Abastecimento. Regras para análise de sementes. Brasília: Mapa/ACS. 399p.

Brito, N.M. Nascimento, L.C.; Coelho, M.S.E. \& Félix, L.P. (2010) Efeitos de óleos essenciais na germinação de sementes de Cereus jamacaru. Revista Brasileira de Ciências Agrárias 5(2): $207-$ 211. DOI: 10.5039/Agraria. v5i2a702

Ellis, M.B. (1971). Dematiaceous Hyphomycetes. Kew. England: Cab International.

Elmer, W.H. (2001). Seeds as vehicles for pathogen importation. Biological Invasios 3 (3): 263-271.

Fernandes, P.C.; Facanali, R.; Teixeira, J.P.F.; Furlani, P.R. \& Marques, M.O.M. (2004). Cultivo de manjericão em hidroponia e em diferentes substratos sob ambiente protegido. Horticultura Brasileira 22 (2): 260-264.

Flávio, N.S.D.S.; Sales, N.L.P.; Aquino, C.F.; Soares, E.P. S.; Aquino, L.F.S. \& Catão, H.C.R.M. (2014). Qualidade sanitária e fisiológica de sementes de sorgo tratadas com extratos aquosos e óleos essenciais. Semina: Ciências Agrárias 35 (1): 7-20. DOI: $10.5433 / 1679-0359.2014 v 35 \mathrm{n} 1 \mathrm{p} 7$

Garcia, R.A.; Juliatti, F.C.; Barbosa, K.A.G. \& Cassemiro, T.A. (2012). Atividade antifúngica de óleo e extratos vegetais sobre Sclerotinia sclerotiorum. Bioscience Journal 28 (1): 48-57.

Gomes, R.; Nunes, M.; Nascimento, L.; Souza, J. \& Porcino, M. (2016). Efficiency of essential oils in the sanitary and physiological quality of lima bean 
seeds (Phaseolus lunatus L.). Revista Brasileira de Plantas Medicinais, 18(1), 279-287.

Hillen, T.; Schwan-Estrada, K.R.F.; Mesquini, R.M.; Cruz, M.E.S.; Stangarlin, J.R. \& Nozaki, M. (2012). Atividade antimicrobiana de óleos essenciais no controle de alguns fitopatógenos fúngicos in vitro e no tratamento de sementes. Revista Brasileira de Plantas Medicinais 14(3):439-445.

Kruppa, P.C. \& Russomanno, O.M.R. (2008). Ocorrência de fungos em sementes de plantas medicinais. aromáticas e condimentares da família Lamiaceae. Tropical Plant Pathology 33(1): 072075. https://doi.org/10.1590/S1982-

56762008000100013 .

Kruppa, P.C. \& Russomanno, O.M.R. (2001) Fungos associados à sementes de manjericão (Ocimum basilicum). Arquivos do Instituto Biológico 68(Supl.):57.

Kruppa, P.C. \& Russomanno, O.M.R. (2011). Fungos em plantas medicinais. aromáticas e condimentares - solo e sementes. Biológico 73(1): 33-38.

Kruppa, P.C. \& Russomano, O.M.R. (2009). Fungos em plantas medicinais, aromáticas e condimentares solo e semente. 2009. Artigo em Hypertexto. Disponível em: <http://www.infobibos.com/Artigos/2009_1/Fungos/ index.htm>. Acesso em: 27/2/2020.

Lima, C.B. \& Villela, T.T. (2017). Efeito dos óleos essenciais de alho e laranja e do surfactante Tween ${ }^{\circledR 80}$ sobre a germinação de sementes de manjericão. Revista de Ciências Agroambientais 15(2):83-91.

Lopes, J.E.L.; Santos, M.A.M.; De Oliveira, A.L.T.; Pinheiro, J.V. \& Bezerra, A.M.E. (2013) Comparação dos tratamentos sol pleno e casa de vegetação no crescimento de (Copaifera langsdorffii Desf). Revista Brasileira de Higiene e Sanidade Animal 7(1): 9-21.

Medeiros, J.G.F.; Araújo Neto. A.C.; Medeiros. D.S.; Nascimento. L.C. \& Alves. E.U. (2013).

Extratos vegetais no controle de patógenos em sementes de Pterogyne nitens Tul. Floresta e Ambiente 20(3):384 -390. https://doi.org/10.4322/floram.2013.029.

Medeiros. J.G.F.; Leite, R.P. \& Nascimento, L.C. (2011). Extratos vegetais e seus efeitos na sanidade e fisiologia de sementes de flamboyant -mirim (Caesalpinia pulcherrima L.). In: Seabra. G.; Mendonça. I. (Eds.). Educação ambiental: Responsabilidade para a conservação da sociobiodiversidade. João Pessoa: Editora Universitária. 2011. p. 373- 377.

Medice, R.; Alves, E.; Assis, R.T.D.; Magno Júnior, R. G. \& Lopes, E.A.D.G.L. (2007). Óleos essenciais no controle da ferrugem asiática da soja Phakopsora pachyrhizi Syd. \& P. Syd. Ciência e agrotecnologia, 31(1), 83-90.

Mendes, M.A.S.; Silva, V.L.; Dianese, J.C.;Ferreira, M.A.S.V. ;Santos, C.E.N.; Gomes Neto, E.; Urben, A.F.; Castro ,C. (1998). Fungos em Plantas no Brasil. Brasília DF. Embrapa.

Mendes, M.A.S; Vieira, R.F.; Oliveira, A.S. \& Santos, J.K.P. (2003). Murcha de Fusarium em manjericão no DF. Fitopatologia Brasileira 28 (Supl.):219-220.

Miranda, C.A.S.F.; Cardoso, M. G.; Batista, L.R.; Rodrigues, L.M.A. \& Figueiredo, A.C.S. (2016). Óleos essenciais de folhas de diversas espécies: propriedades antioxidantes e antibacterianas no crescimento espécies patogênicas. Revista Ciência Agronômica $47(1): \quad 213-220 . \quad 2016$. https://doi.org/10.5935/1806-6690.20160025.

Moreau, J.S. (2011) Germinação de sementes em diferentes substratos e caracterização morfológica de plântulas de Anadenanthera macrocarpa (Benth.) Brenan. Monografia (Graduação em Agronomia). Universidade Federal do Espírito Santo. Alegre, ES p. 45.

Ootani, M.A.; Aguiar, R.W.; Ramos, A.C.; Brito, D.R.; Silva, J.B.D. \& Cajazeira, J.P. (2013). Use of essential oils in agriculture. Journal of biotechnology and biodiversity, 4(2), 162-175.

Reis, A.; Miranda, B.E.C.; Boiteux, L.S. \& Henz, G.P. (2007). Murcha do manjericão (Ocimum 
basilicum) no Brasil: agente causal, círculo de plantas hospedeiras e transmissão via semente. Summa Phytopathologica 33(2): 137-141.

Ribeiro, D.S.; Melo, D.B.; Guimarães, A.G. \& Velozo, E.S. (2012). Evaluation of the Rosemary essential oil (Rosmarinus officinalis L.) as modulator of bacterial resistance. Semina: Ciências Agrárias 33(2): 687-696. http://dx.doi.org/10.5433/1679$0359.2012 \mathrm{v} 33 \mathrm{n} 2 \mathrm{p} 687$

Rosado, L.D.S.; Pinto, J.E.B.P.; Botrel, P.P.; Bertolucci, S.K.V.; Niculau, E.S. \& Alves, P.B. (2011) Influência do processamento da folha e tipo de secagem no teor e composição química do óleo essencial de manjericão cv. Maria Bonita. Ciência e Agrotecnologia 35(2): 291-296.

Russomanno, O.M.R.; Kruppa, P.C. Martins, A. \& Figueiredo, M.B. (2004). Murcha de Fusarium oxysporum em plantas de alecrim (Rosmarinus officinalis) e manjericão anão (Ocimum minimum). Summa Phytopathologica 30:98. (Resumo)

Russomanno, O.M.R. \& Kruppa, P.C. (2010). Doenças fúngicas das plantas medicinais, aromáticas e condimentares - parte aérea. Biológico (Brasil) 72:31-37.

Sales, N. L. P.; Cota, C.G.; Freitas, F.G.R.; Moreira, J.L.; Carvalho, L.R. Moreira, C.D.D. \& Barroso, P.D. (2018) Germinação, sanidade e tratamento de sementes de Guazuma ulmifolia Lam. Caderno de Ciências Agrárias 10(2): 46-52.
Sarmento-Brum. R.B.C.; Santos. G.R.; Castro. H.G.; Gonçalves. C.G.; Cardon. C.H.; Leão. E.U. \& Sarmento. R.A. (2013). Avaliação in vitro de diferentes métodos de análises de fungitoxicidade de óleos essenciais. Bioscience Journal 29(3): 623626.

Seixas. P.T.L.; Castro. H.C.; Santos. G.R. \& Cardoso. D.P. (2011). Controle fitopatológico do Fusarium subglutinans pelo óleo essencial do capim citronela (Cymbopogon nardus L.) e do composto citronelal. Revista Brasileira de Plantas Medicinais 13: 523-526.

Silva. J.A.; Pegado. C.M.A. \& Ribeiro. V.V. (2009) Efeito de extratos vegetais no controle de Fusarium oxysporum $f$. $\mathrm{sp}$. tracheiphilum em sementes de caupi. Ciência e Agrotecnologia 33(2):611-616.

Silva, C. T., Franco, T. D. \& Jordão, C. O. (2016). Análise fitoquímica e ensaio toxicológico do extrato hidroalcoólico de Morinda citrifolia (NONI). Revista Brasileira Multidisciplinar, 19(1), 34-39.

Piveta, G.; Menezes, V.O.; Pedroso, D.C.; Muniz, M.F.B.; Blume, E. \& Wielewicki, A.P. (2010). Superação de dormência na qualidade de sementes e mudas: influência na produção de Senna multijuga (L. C. Rich.) Irwin \& Barneby. Acta Amazônica 40(2): 281-288. https://doi.org/10.1590/S004459672010000200006

Urben, A.F.; Mattos, J.K.A. \& Mendes, M.A.S. (1987). Fungos associados a manchas de folhas em plantas de uso medicinal no Distrito Federal. Fitopatologia Brasileira 12:390-394. 